\title{
Comparison of remote ischemic preconditioning and intermittent hypoxia training in fracture healing
}

\author{
JUNJIE QIAO ${ }^{1}$, MENG ZHOU ${ }^{2}$, ZHENG LI $^{1}$, JIE REN $^{1}$, GUANGHAN GAO $^{1}$, \\ GUANGLEI CAO $^{1}$, HUILIANG SHEN ${ }^{1}$ and SHIBAO LU ${ }^{1}$ \\ ${ }^{1}$ Department of Orthopedics, Xuanwu Hospital, Capital Medical University, Beijing 100053; ${ }^{2}$ Department of Orthopedics, \\ Beijing Jishuitan Hospital, 4th Medical College of Peking University, Beijing 100035, P.R. China
}

Received May 30, 2018; Accepted November 7, 2018

DOI: $10.3892 / \mathrm{mmr} .2018 .9788$

\begin{abstract}
Fracture healing in elderly patients is an emerging public health concern. As non-drug treatments, intermittent hypoxia training (IHT) and remote ischemic preconditioning (RIPC) are considered to have substantial advantages and to aid fracture healing in elderly patients. The purpose of the present study was to evaluate and compare the effects of IHT and RIPC on fracture healing. Micro-computed tomography (micro-CT) and biomechanical testing were used to assess the morphology and structural properties of bone callus dissected from aged rats with tibial fractures. In addition, hypoxia-inducible factor- $1 \alpha$ (HIF-1 $\alpha)$ and its target gene, associated with the healing process, were investigated by reverse transcription-quantitative polymerase chain reaction and western blot analyses. The micro-CT-based parameters, including bone mineral density and trabecular number, were measured, and significant differences were identified between the experimental and control groups. The IHT group exhibited superior bone formation and mineralization rates compared with the RIPC group. The biomechanical testing revealed that the ultimate loading and stiffness values were significantly higher in the IHT group compared with those in the RIPC group. In accordance with previous studies, RIPC exerted a similar effect in increasing the expression of HIF-1 $\alpha$, and its
\end{abstract}

Correspondence to: Professor Huiliang Shen or Dr Guanglei Cao, Department of Orthopedics, Xuanwu Hospital, Capital Medical University, 45 Changchun Street, Beijing 100053, P.R. China

E-mail: dr_shenhuiliang@sina.com

E-mail: guanglei_cao@163.com

Abbreviations: IHT, intermittent hypoxia training; RIPC, remote ischemic preconditioning; HIF-1 $\alpha$, hypoxia-inducible factor- $1 \alpha$; BV, bone volume; $\mathrm{BV} / \mathrm{TV}$, total bone/total volume; $\mathrm{BMD}$, bone mineral density; Tb.N, trabecular number; ALP, alkaline phosphatase; OCN, osteocalcin; Runx2, runt-related transcription factor 2; VEGF, vascular endothelial growth factor; Micro-CT, micro-computed tomography; RT-qPCR, reverse transcription-quantitative polymerase chain reaction

Key words: remote ischemic preconditioning, intermittent hypoxia training, hypoxia induced factor- $1 \alpha$, fracture healing downstream genes, throughout the course of healing. In addition, the IHT group exhibited increased expression levels of HIF-1 $\alpha$ compared with the RIPC group. Taken together, the results suggested that IHT and RIPC significantly enhanced fracture healing; however, IHT exhibited superior bone formation and healing effects compared with RIPC.

\section{Introduction}

Considering the rapidly increasing aging population, the number of elderly patients with fractures is likely to rise in the near future (1). It has been previously reported that the risk of non-union increases with age and fracture in aged patients is associated with high morbidity and mortality rates, in addition to increased healthcare costs (2). Fracture healing in aged patients is an emerging public health concern, and how to promote fracture healing has been investigated extensively in recent decades.

Remote ischemic preconditioning (RIPC) is the process of inducing interspersed cycles of ischemia in a remote organ to prevent ischemic damage of the target organ. In previous years, RIPC has emerged as an innovative and successful therapeutic procedure for ischemia and reperfusion (3). Recently, it has been revealed to be effective in fracture healing in a rat model (4).

Compared with RIPC, intermittent hypoxia training (IHT) has a long developmental history and there is a wealth of information regarding its application in the biomedical field (5). It has been demonstrated that IHT has a marked effect on increasing resistance to severe hypoxia/ischemia (6). Fractures that disrupt the blood supply and isolate the damaged bone from perfusion cause low oxygen tension and regional hypoxia (7). Therefore, it was hypothesized that IHT may be critical in fracture healing. RIPC and IHT may become widespread therapies due to their ease of access and effectiveness; therefore, a comprehensive understanding of IHT and RIPC is required to reduce potential harmful consequences and to maximize potential utility in fracture healing.

The present study aimed to provide insight into the role of IHT in fracture healing and the differing healing effects of IHT and RIPC. To the best of our knowledge, this is the first systematic study to compare the effects of IHT and RIPC in fracture healing of the aged. It was hypothesized that IHT 
may have a positive effect on fracture healing and that the application of IHT may enhance bone healing. Furthermore, compared with RIPC, enhanced healing results may be achieved through the use of IHT.

\section{Materials and methods}

Animal care and establishment of the fracture model. All experimental procedures involving rats were approved by the Ethics Committee on Animal Experimentation of Capital Medical University (Beijing, China; no. AEEI-2017-098). A total of 96 male Sprague-Dawley rats $(485 \pm 60 \mathrm{~g})$ were obtained from the Laboratory Animal Center of Capital Medical University. In preliminary experiments, 22-24-month-old rats were used. However, the drop in weight usually resulted in a comminuted fracture of the tibia and the number of rats with comminuted-fractures decreased significantly when 18-20-month-old rats were used. In addition, others have reported the use of 18-20-month-old rats as aged rats $(8,9)$, therefore, $18-20$-month-old rats were used in the present study. The animals were housed at a temperature of $23-25^{\circ} \mathrm{C}$, humidity of $50-60 \%$ and with unlimited access to food and water. Upon arrival at the central animal facility, the rats were allowed to acclimatize for 1 week prior to being randomly assigned to three groups: IHT $(n=32)$, RIPC $(n=32)$ and control $(n=32)$. RIPC was initiated immediately following surgery by occluding blood flow in the contralateral hind limb. Hind limb occlusion was performed by three cycles of tightening and releasing of a tourniquet $(18-\mathrm{mm})$ around the upper thigh, with each occlusion or the release phase lasting $10 \mathrm{~min}$. This method has been previously demonstrated to completely occlude the blood flow, as assessed using the vascular assessments system (Periflux System 5000; Perimed AB, Järfälla, Sweden) (4). The procedure was performed once a day for 28 consecutive days. The IHT training was performed in a normobaric hypoxic cabin constructed by the Hypoxia Research Center of Xuanwu Hospital (Beijing, China). The rats were placed in the cabin immediately following surgery and the IHT regimen lasted 5 min with $12 \% \mathrm{O}_{2}$ and 5 -min breaks. A total of five cycles were performed per day, and the control group underwent surgery only.

Subsequent to peritoneal injection of sodium pentobarbital (40 $\mathrm{mg} / \mathrm{kg}$ body weight), fracture of the left tibia was achieved with a blunt guillotine apparatus driven by a drop weight, as previously described (4). To achieve intramedullary fixation, a $0.8-\mathrm{mm}$ Kirschner-wire (K-wire) was inserted through the intercondylar notch until it was seated in the distal cortex. Radiographs were obtained immediately to confirm K-wire placement and the extent of the fractures. Comminuted fractures or crack fractures of the tibia were excluded from the study. The rats were sacrificed by cervical dislocation following the same administration of the anesthetic described above at 7,14 and 28 days post-fracture $(n=8$ at each time point).

Following removal of the K-wires, the tibiae were dissected and prepared for reverse transcription-quantitative polymerase chain reaction (RT-qPCR) analysis, western blotting, micro-computed tomography (micro-CT) and biomechanical testing.
Micro-CT analysis. A Siemens inveon micro-CT scanner (Siemens AG, Munich, Germany) was used to scan the dissected tibia, and the K-wires were removed carefully in order to protect the fracture site. The beam protocol was set as follows: $15-\mu \mathrm{m}$ isometric voxel size, $800 \mathrm{~mA}$ and $80 \mathrm{kV}$. The proximal and distal bone tissues $5 \mathrm{~mm}$ from the fracture line were selected as regions of interest (ROI). The callus perimeter was determined using a semi-automated contouring method. Contours were drawn to reveal the periosteal surface of the ROI in the tibia. Mimics software version 20.0 (Materialise $\mathrm{NV}$, Leuven, Belgium) was used for the three-dimensional reconstruction of the tibia. The following bone structural parameters were measured and statistically analyzed by the internal software of the micro-CT system: Bone volume $\left(\mathrm{BV}, \mathrm{mm}^{3}\right)$, bone volume/total volume $(\mathrm{BV} / \mathrm{TV}, \%)$, bone mineral density (BMD, mg $\mathrm{HA} / \mathrm{cm}^{3}$ ) and trabecular number (Tb.N, 1/mm).

Biomechanical testing. Three-point bending tests were applied to evaluate the biomechanical properties of the fracture site. The biomechanical tests were performed at room temperature using a testing apparatus (ELF 3400; Enduratec Systems Group; Bose Corporation, Framingham, MA, USA) with the distance between the rollers set as $20-\mathrm{mm}$. Following careful removal of the K-wire, all specimens were subjected to an axial compressive force $(2 \mathrm{~mm} / \mathrm{min})$ until fracture occurred. The applied forces and resulting displacements were recorded. The stiffness $(\mathrm{N} / \mathrm{mm}$, the slope of the linear portion of the load-deformation curve) and ultimate loading ( $\mathrm{N}$; the maximum force that the specimen sustained) were calculated.

RNA extraction and RT-qPCR analysis. Total RNA was isolated from the callus tissue using TRIzol according to the manufacturer's protocol (Invitrogen; Thermo Fisher Scientific, Inc., Waltham, MA, USA), and then reverse transcribed into the cDNA) as following step: $42^{\circ} \mathrm{C}$ for $20 \mathrm{~min}$, then $99^{\circ} \mathrm{C}$ for 5 min using the ReverTra Ace kit (Toyobo Co., Ltd., Osaka, Japan). The reacted solution was stored at $-20^{\circ} \mathrm{C}$. RT-qPCR analysis was performed to measure mRNA expression levels relative to the expression of GAPDH using an icycle iq real-time PCR detection system (Bio-Rad laboratories, Inc., Hercules, CA, USA) using SYBR-Green Master mix. A total of $1 \mu \mathrm{l}$ of cDNA $(10 \mathrm{ng} / \mu \mathrm{l})$ was used for qPCR analysis in a $9 \mu \mathrm{l}$ reaction volume with $5 \mu \mathrm{l}$ of SYBR-Green Master mix, $1 \mu \mathrm{l}$ of forward primer ( $1 \mathrm{umol} / \mathrm{l}), 1 \mu \mathrm{l}$ of reverse primer ( $1 \mu \mathrm{mol} / \mathrm{l}), 2 \mu \mathrm{l}$ of $\mathrm{ddH}_{2} \mathrm{O}$. The sequences of the primers used in the present study were as follows: Runt-related transcription factor 2 (Runx2) forward, 5'-CCCACGAATGCACTATCC AG-3' and reverse, 5'-GGCTTCCATCAGCGTCAACA-3'; alkaline phosphatase (ALP) forward, 5'-GGACGGTGAACG GGAGAAC-3' and reverse, 5'-CCCTCAGAACAGGGTGCG TAG-3'; hypoxia-inducible factor-1 $\alpha$ (HIF-1 $\alpha$ ) forward, 5'-CCC CTACTATGTCGCTTTCTTGG-3' and reverse, 5'-GGTTTC TGCTGCCTTGTATGG-3'; vascular endothelial growth factor (VEGF) forward, 5'-CGACAAGGCAGACTATTC AACG-3' and reverse, 5'-GGCACGATTTAAGAGGGG AAT-3'; osteocalcin (OCN) forward, 5'-CGGACCACATTG GCTTCCAG-3' and reverse, 5'-GCTGTGCCGTCCATACTT TCG-3'; GAPDH forward, 5'-TGACAACTTTGGCATCGT GG-3' and reverse, 5'-GGGCCATCCACAGTCTTCTG-3'. 
A
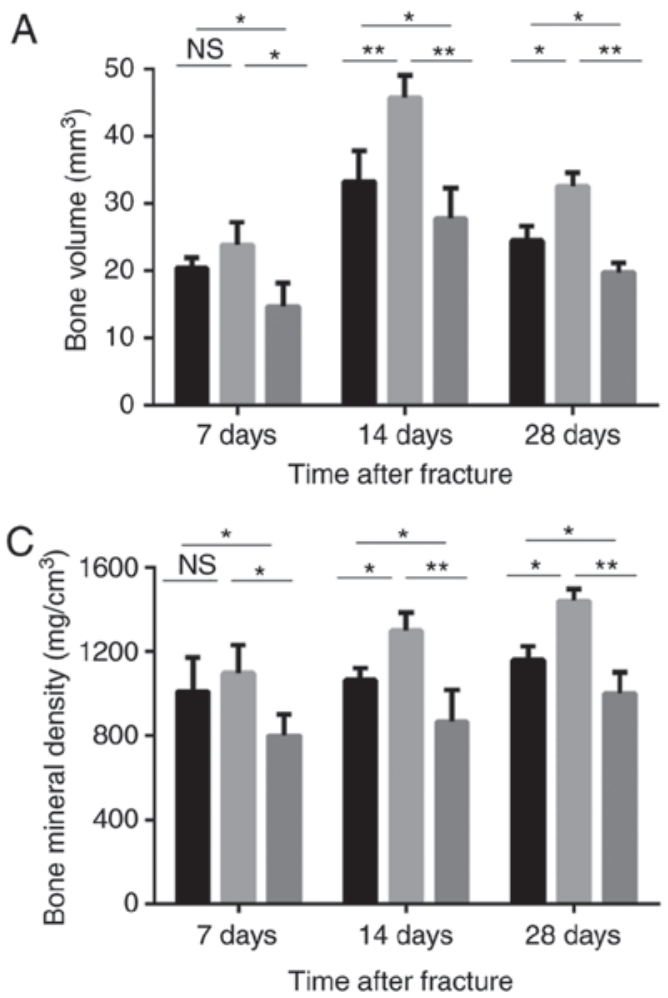

$\mathrm{B}$
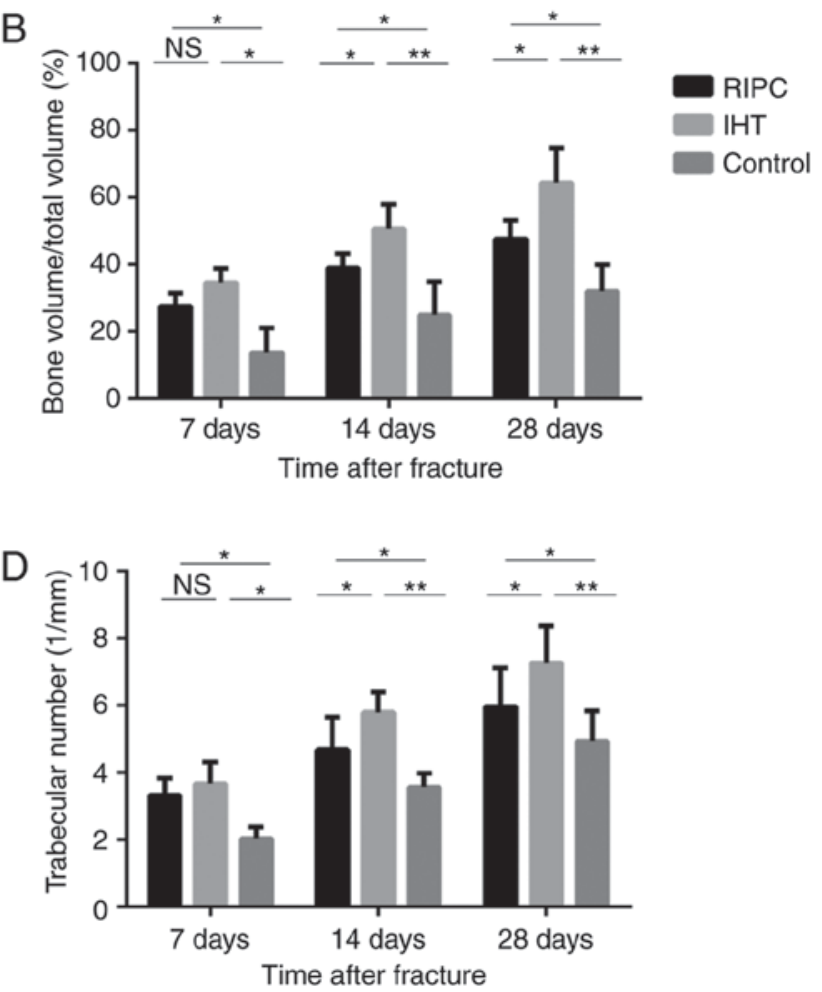

Figure 1. Comparison of structural parameters using micro-computed tomography among the IHT, RIPC and control groups. Compared with the RIPC group, IHT accelerated the formation of bone callus and the subsequent remodeling process. (A) Bone volume, (B) total bone volume fraction, (C) bone mineral density and (D) trabecular numbers. Values are presented as the mean \pm standard deviation, ${ }^{*} \mathrm{P}<0.05,{ }^{* *} \mathrm{P}<0.01$, N.S., not significant; IHT, intermittent hypoxia training; RIPC, remote ischemic preconditioning.

The thermocycling conditions were as follows: $95^{\circ} \mathrm{C}$ for $5 \mathrm{~min}$ followed by 40 cycles of $95^{\circ} \mathrm{C}$ for $15 \mathrm{sec}$ and $60^{\circ} \mathrm{C}$ for $1 \mathrm{~min}$. The expression values were normalized to GAPDH using the $2^{-\Delta \Delta \mathrm{Cq}}$ method (10). In order to minimize confounding variance, two independent samples were analyzed three times. Technical replicates were averaged prior to all software analysis.

Western blotting. The dissected callus tissues from fractured tibia at each stage (7, 14 and 28 days) were washed with PBS and ground into a powder in liquid nitrogen, following which the tissue lysates were prepared with RIPA buffer containing protease inhibitors (Sigma-Aldrich; Merck KGaA, Darmstadt, Germany). The protein concentration was determined using a bicinchoninic acid protein assay kit (Thermo Fisher Scientific, Inc.). The lysates (30 mg) were separated on $10 \%$ SDS-polyacrylamide gels and transferred onto polyvinylidene difluoride membranes (EMD Millipore, Bedford, MA, USA). The membranes were blocked with 5\% non-fat dry milk for $1 \mathrm{~h}$ at room temperature and incubated overnight at $4^{\circ} \mathrm{C}$ with the following primary antibodies: ALP (cat. no. ab84401; 1:2,000; Abcam, Cambridge, UK), Runx2 (cat. no. H00000860-M04; 1:500; Abnova, Taipei, Taiwan), HIF-1 $\alpha$ (cat. no. ab463; 1:1,000), VEGF (cat. no. ab46154; $1: 1,000)$, OCN (cat. no. ab13420; 1:1,000), GAPDH (cat. no. ab8245; 1:1,000; all Abcam). This was followed by incubation for $1 \mathrm{~h}$ with a horseradish peroxidase-conjugated secondary antibody (cat. no. 7076P2; 1:5,000; Cell Signaling Technology, Inc, Danvers, MA, USA) at $37^{\circ} \mathrm{C}$. The bands were developed using chemiluminescence (Thermo Fisher Scientific, Inc.) and the densitometric results were analyzed with Image Quant LAS4000 software (GE Healthcare Life Sciences, Little Chalfont, UK). GAPDH was used as a loading control.

Statistical analysis. Each experiment was repeated for three times. All statistical analyses were performed with SPSS software, version 19.0 (IBM Corp., Armonk, NY, USA). Data are presented as the mean \pm standard deviation. The differences between groups were analyzed by one-way analysis of variance followed by Dunnett's test. $\mathrm{P}<0.05$ was considered to indicate a statistically significant difference.

\section{Results}

IHT accelerates callus formation and the subsequent remodeling process in fracture healing. Quantitative analysis of callus formation by micro-CT is presented in Fig. 1A-D. The callus tissues formed in the experimental groups were significantly different from those in the control group at all time points. Although the callus in the IHT group exhibited a larger BV than that in the RIPC group, no significant difference was observed in any parameter at 7 days. At 14 days post-fracture, all parameters were increased. At this time-point, the IHT group exhibited significantly higher BV, BMD, BV/TV and Tb.N values than in the RIPC group. At 28 days post-fracture, the BV of the IHT group was markedly decreased compared with that of the RIPC group, whereas the other parameters continued to increase. In addition, higher BMD, BV/TV and Tb.N values were recorded in the IHT group than the RIPC group. As low BV and high BMD are representative of bone reconstruction in fracture healing, IHT 
A
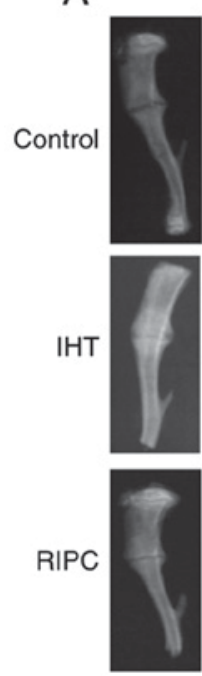
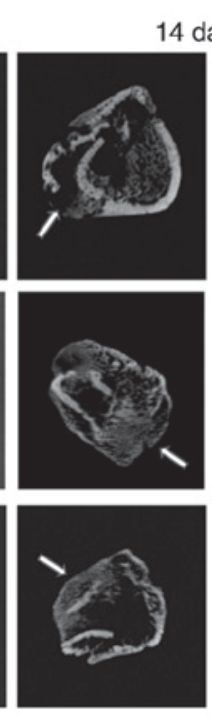

14 days
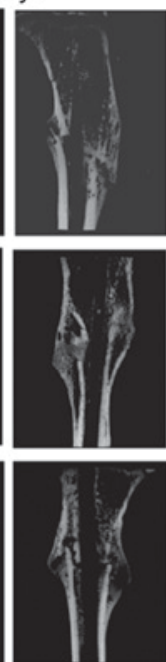

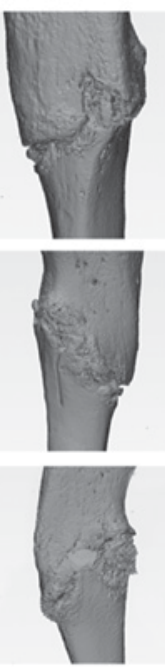

B
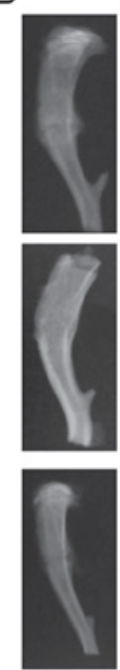
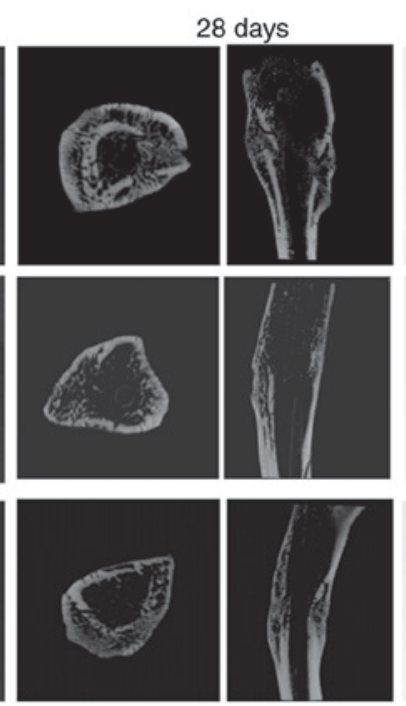

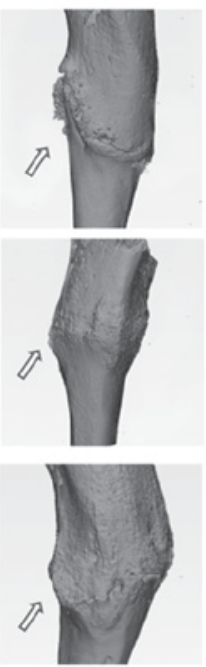

Figure 2. Representative visualization of tibiae at 14 and 28 days. (A) Images of fractured tibiae at 14 days post-fracture. (B) Images of fractured tibiae at 28 days post-fracture. The upper image is representative of the control group, the middle image is representative of the IHT group and the lower image is representative of the RIPC group. Images from left to right indicate plain radiographic, coronal plane, sagittal plane and three-dimensional reconstruction images of the bone callus around the fracture line, respectively. White arrows show callus formation and the fracture line in A and B, respectively. IHT, intermittent hypoxia training; RIPC, remote ischemic preconditioning.
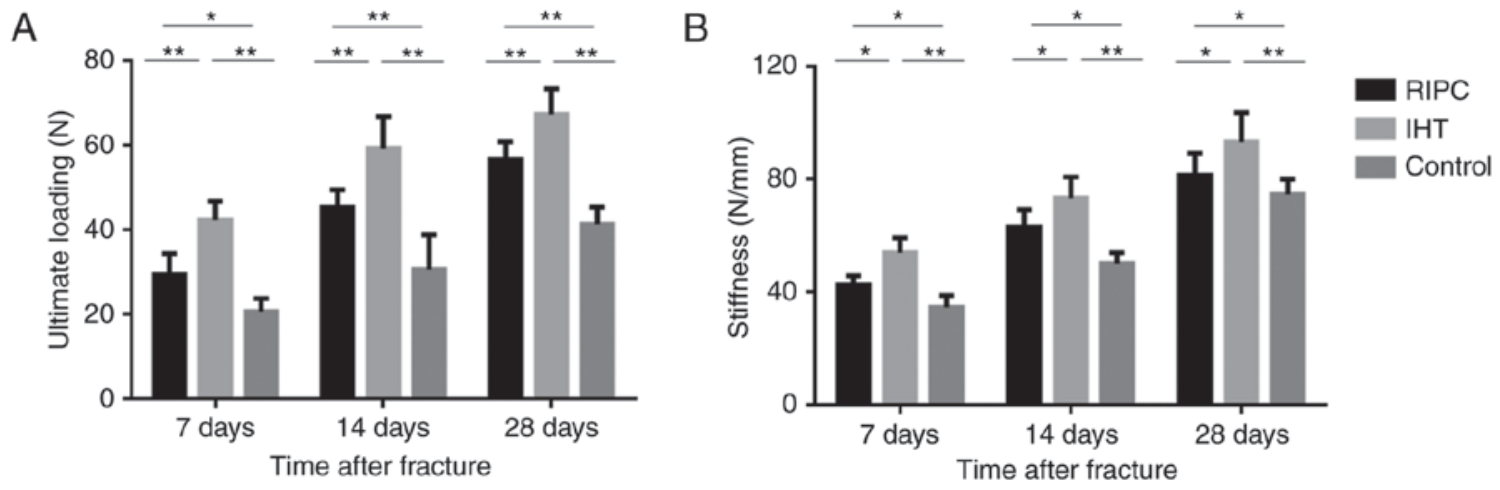

Figure 3. Effects of IHT and RIPC on the biomechanical properties of dissected tibiae at all time points, measured using a three-point bending test. The IHT group exhibited superior biomechanical properties compared with the other groups at 14 and 28 days post-fracture. (A) Statistical analysis of ultimate loading, (B) Statistical analysis of stiffness. Values are presented as the mean \pm standard deviation. ${ }^{*} \mathrm{P}<0.05,{ }^{* *} \mathrm{P}<0.01$. IHT, intermittent hypoxia training; RIPC, remote ischemic preconditioning.

may promote fracture healing by accelerating callus formation and the subsequent remodeling process.

$\mathrm{X}$-ray and three-dimensional reconstructions of the tibiae are presented in Fig. $2 \mathrm{~A}$ and $\mathrm{B}$, which provide a more intuitive delineation of the results. The experimental groups exhibited more bridging callus at the fracture site compared with the control group at 14 days. The IHT group demonstrated loss of the fracture line at 28 days, whereas the RIPC group exhibited dense bone callus around the fracture line. These results indicated that the healing effect of IHT was more efficient than that of RIPC at all time points.

IHT improves the biomechanical properties of the fractured tibia. The ultimate loading and stiffness values are presented in Fig. 3A and B. From a biomechanical perspective, the ultimate loading and stiffness values are considered to be measures of resistance to failure and deformation of the tibia. The experimental groups exhibited enhanced biomechanical properties compared with the control group in terms of ultimate loading and stiffness, at all time points $(\mathrm{P}<0.05)$. In addition, the ultimate loading and stiffness values were significantly higher in the IHT group than in the RIPC group.

\section{IHT promotes osteoblast differentiation and mineralization.} Osteoblasts are involved in the process of fracture healing; therefore, the present study analyzed the expression of osteoblast markers among the three groups, including VEGF, Runx2, ALP and OCN. The results of the western blot (Figs. 4 and 5A-D) and RT-qPCR (Fig. 6A-D) analyses showed that the mRNA and protein expression levels of VEGF, Runx2, ALP and OCN were upregulated in the IHT and RIPC groups at all time points. Furthermore, compared with the RIPC group, the IHT group exhibited higher expression of all markers at all time points. These results indicate that IHT may have a positive effect on osteoblast differentiation, which promotes early fracture healing. 


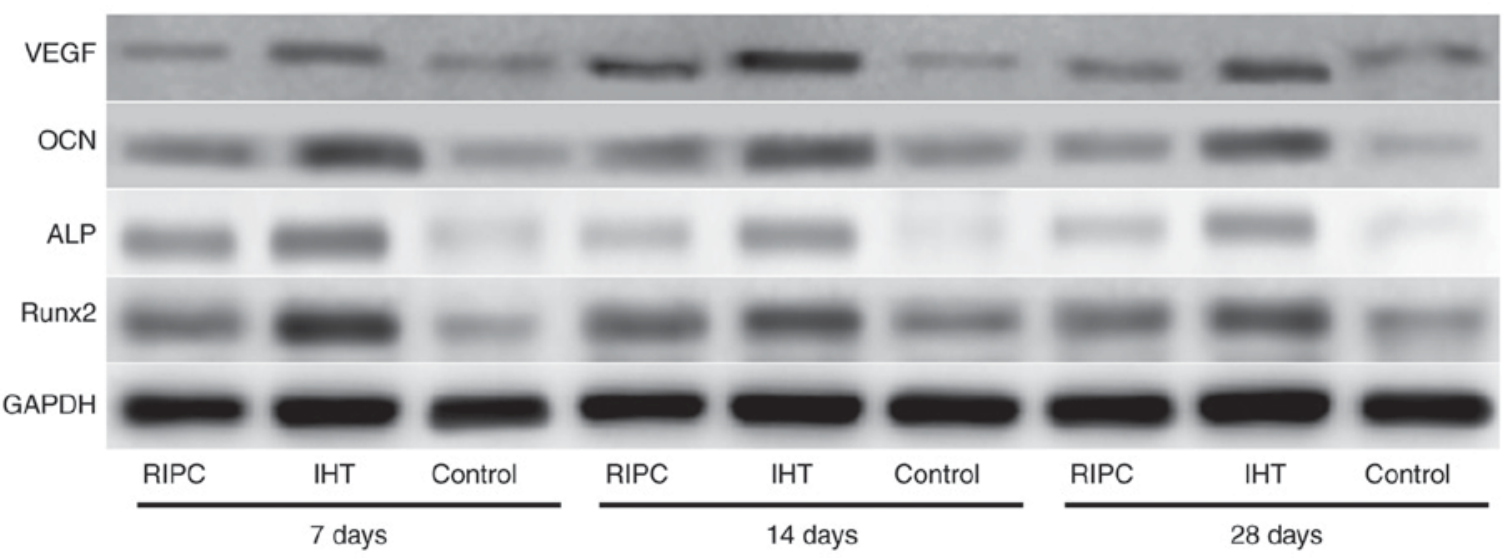

Figure 4. Expression levels of VEGF, OCN, ALP and Runx 2 in bone callus tissues of the IHT, RIPC and control groups, analyzed by western blotting. GAPDH was used as an internal control. IHT, intermittent hypoxia training; RIPC, remote ischemic preconditioning; VEGF, vascular endothelial growth factor; OCN, osteocalcin; ALP, alkaline phosphatase; Runx2, runt-related transcription factor 2.
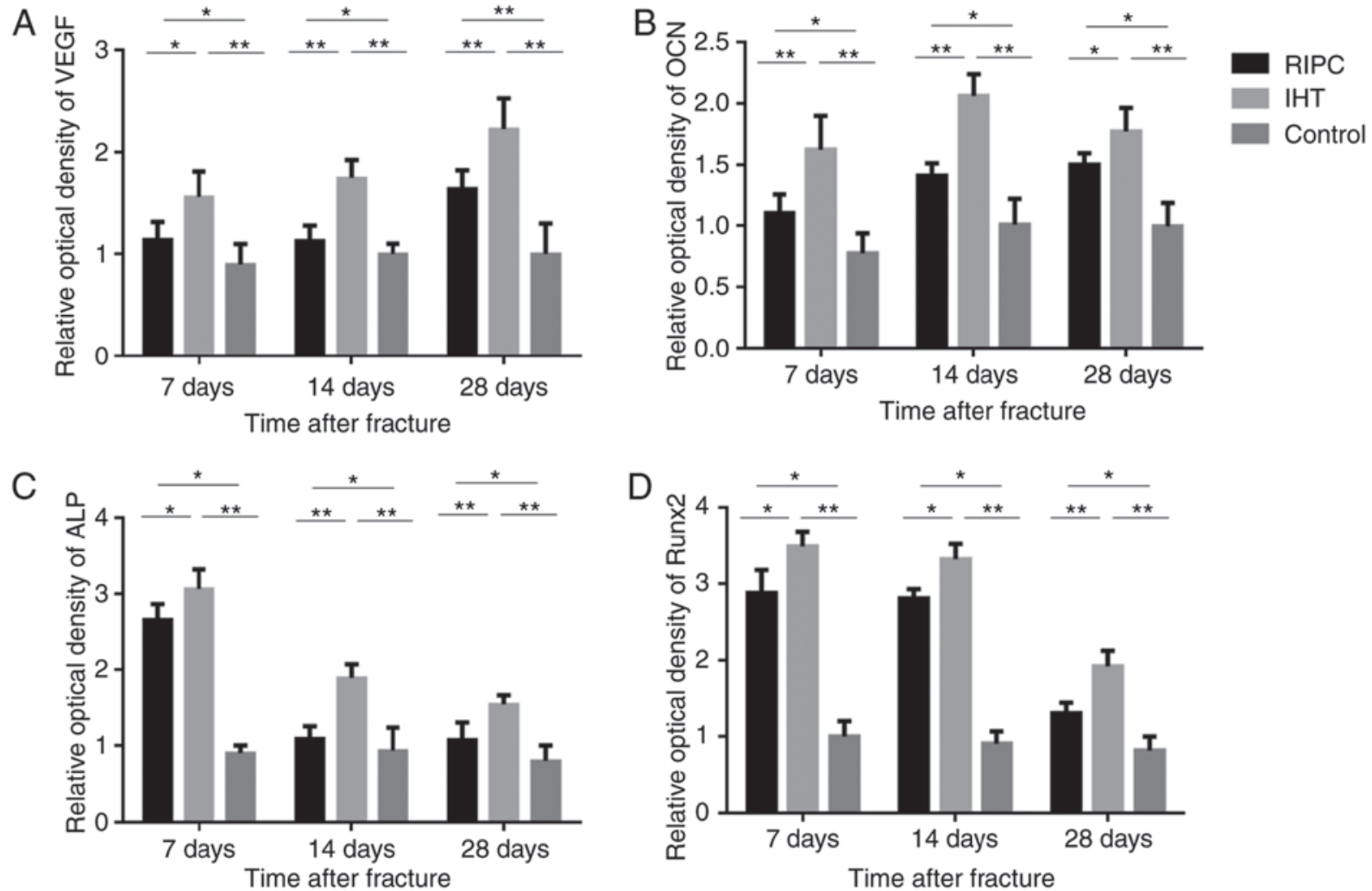

Figure 5. Optical density of (A) VEGF, (B) OCN, (C) ALP and (D) Runx2 in bone callus tissues of the IHT, RIPC and control groups, analyzed by western blotting. GAPDH was used as an internal control. " $\mathrm{P}<0.05,{ }^{\text {** }} \mathrm{P}<0.01$. IHT, intermittent hypoxia training; RIPC, remote ischemic preconditioning; VEGF, vascular endothelial growth factor; OCN, osteocalcin; ALP, alkaline phosphatase; Runx2, runt-related transcription factor 2.

IHT induces the expression of HIF-1 $\alpha$. It has been well established that VEGF, Runx2, ALP and OCN are target genes of HIF-1 $\alpha$. Therefore, to elucidate the mechanism underlying the induction of osteoblast marker expression, the expression of HIF-1 $\alpha$ was also detected by western blot and RT-qPCR analyses. The results showed that the levels of HIF-1 $\alpha$ were significantly increased in all experimental groups compared with levels in the control group at all time points $(\mathrm{P}<0.01)$. Furthermore, the levels of HIF- $1 \alpha$ in the IHT group were significantly increased compared with levels in the in RIPC group (Fig. 7A and B).

\section{Discussion}

The elderly population is increasing, and the repair and regenerative capacity of bone tissue in elderly patients is limited (11). It is also important to consider the type and dosage of medicine administered to elderly patients, due to their decreased drug metabolism (12). Therefore, the identification of a novel method to promote fracture healing in elderly patients is required.

In the present study, the micro-CT findings support the understanding that ITH and RIPC have a positive effects on 

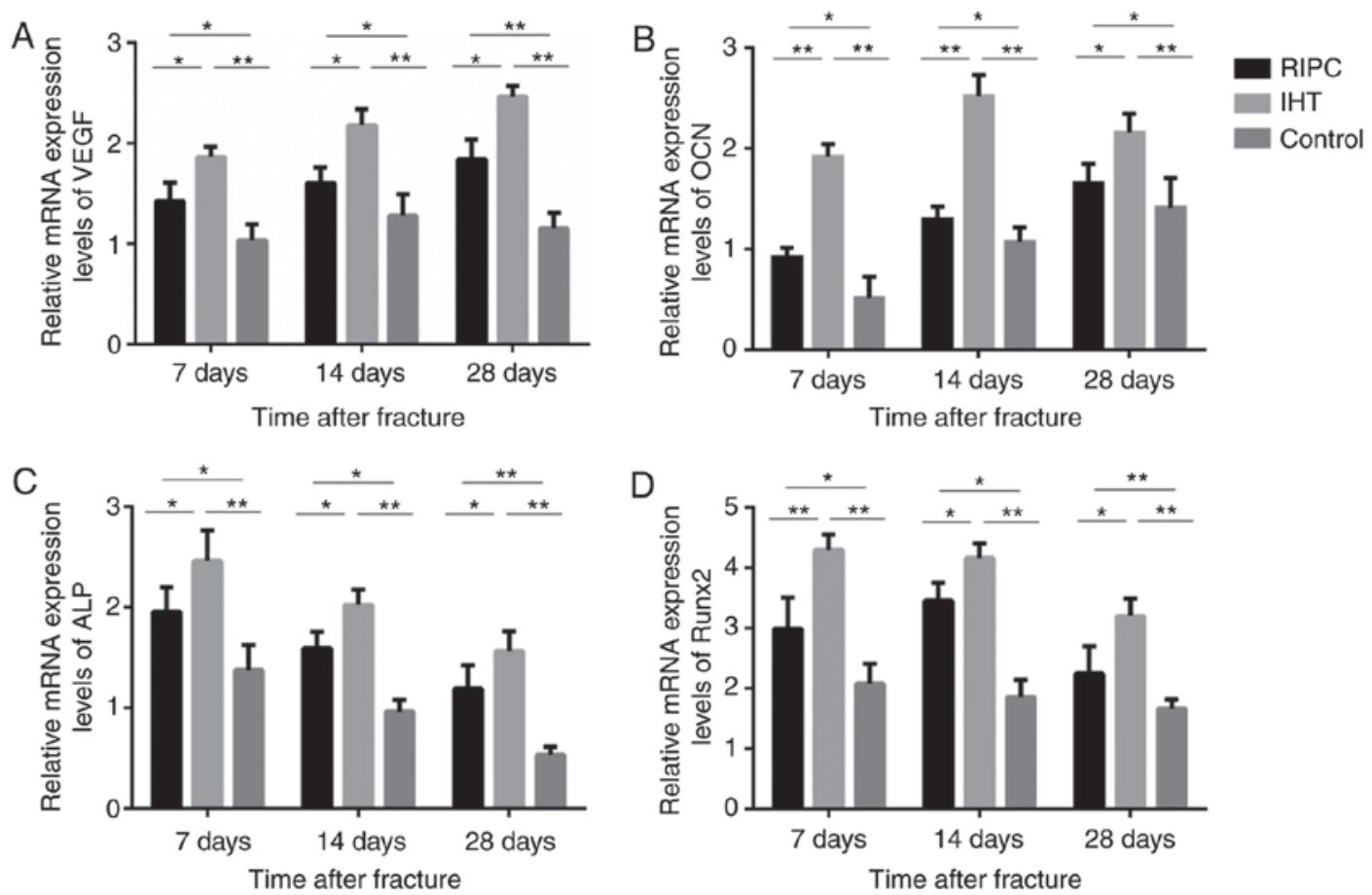

Figure 6. mRNA analysis of VEGF, OCN, ALP and Runx2 in bone callus tissues of the IHT, RIPC and control groups. Expression levels of VEGF, Runx2, ALP and OCN were upregulated in the IHT and RIPC groups, and the IHT group exhibited the highest expression of the markers. Expression levels of (A) VEGF, (B) OCN, (C) ALP, and (D) Runx2, with GAPDH as an internal control. ${ }^{*} \mathrm{P}<0.05$ and ${ }^{* *} \mathrm{P}<0.01$. IHT, intermittent hypoxia training; RIPC, remote ischemic preconditioning; VEGF, vascular endothelial growth factor; OCN, osteocalcin; ALP, alkaline phosphatase; Runx2, runt-related transcription factor 2.

fracture healing. $\mathrm{BV}$, which is sensitive to the detection of changes in the early stage of fracture healing, was significantly increased in the experimental groups compared with the control group, and peaked at 14 days post-fracture (13). Among the other parameters, BMD, which is the most sensitive in detecting changes in healing, increased with time (14). Compared with the RIPC group, BMD and Tb.N were increased in the IHT group in the late stage of fracture healing. Combining these results, it may be interpreted that the amount of mineralized tissue and bone trabecula formed in the RIPC group are lower than those in the IHT group, and that IHT accelerates the remodeling process in fracture healing.

Biomechanical assessment is regarded as the gold standard for evaluating the effects of various interventions on the structural properties of a callus (15). Despite the fact that the RIPC performed poorly compared with the IHT group at a relatively low pressure, the IHT and RIPC groups had significantly higher ultimate loading and stiffness values than the control group. The brittle behavior of the callus in the IHT group is indicative of a high mineralization rate at the fracture site. One possible explanation is that the bony microarchitecture formed during the regenerative process may influence the biomechanical properties of bone directly, and that IHT may accelerate osteoblast proliferation and subsequent mineralization in the early stage of fracture healing.

Fracture healing is a regenerative process that involves distinct temporal and spatial patterning of gene expression among different cell types (16). To further illustrate the molecular mechanisms of IHT and RIPC, the present study detected the levels of ALP, Runx2, OCN and VEGF in the bone callus to investigate the effect of IHT and RIPC on osteoblasts. Runx 2 and OCN are considered to be the primary controlling transcription factors in the early and late stages of osteoblast differentiation, respectively (17). VEGF is critical in the extensive neovascularization of fracture sites, and has been demonstrated to be important in stimulating bone healing (18). ALP is also a representative marker of differentiation, secreted by osteoblasts in response to osteogenic activity. This was confirmed by RT-qPCR and western blot analyses. Furthermore, the IHT group exhibited significantly higher expression of these markers at all time points.

It has been demonstrated that HIF-1 $\alpha$ mainly responds to changes in oxygen levels, and that it is important in adapting to conditions including ischemia and hypoxia. Therefore, HIF-1 $\alpha$ is considered to be involved in IHT and RIPC (19). Consistent with previous studies, the RIPC group exhibited a similar effect of upregulating the expression of HIF-1 $\alpha$ throughout the course of healing, which was demonstrated at the mRNA and protein levels. In addition, the IHT group exhibited higher differential expression levels of HIF-1 $\alpha$ compared with the RIPC group. HIF-1 $\alpha$ is able to interact with the core DNA sequence of the hypoxia response element, resulting in upregulation of the expression of multiple hypoxia-sensitive target genes (20). In the present study, the results suggested that RIPC and IHT increased the expression of HIF-1 $\alpha$ and osteoblast markers. These hypoxia-sensitive genes were further increased in the IHT group compared with the RIPC group, which was in accordance with the micro-CT and biomechanical results. Taken together, it was concluded that RIPC and IHT may promote fracture healing by activating the HIF-1 $\alpha$ pathway. The expression level of HIF-1 $\alpha$ was significantly higher in the IHT group than in the RIPC group, which may explain the superior fracture healing induced by IHT. 
A
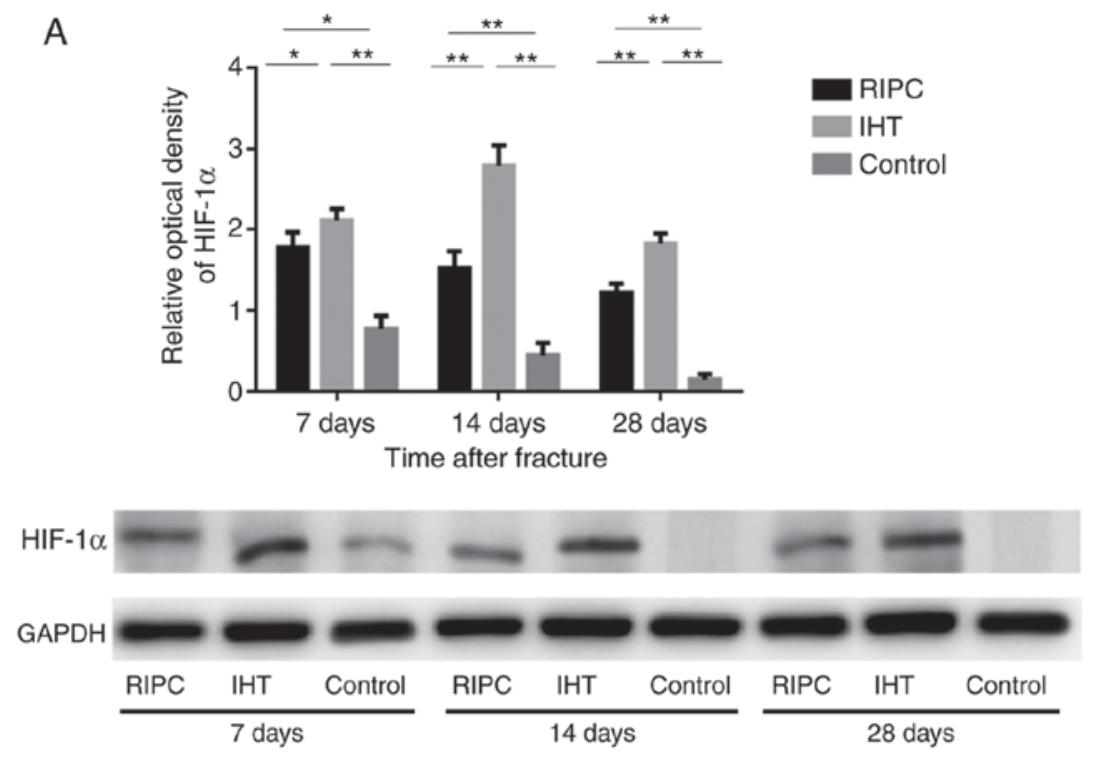

B

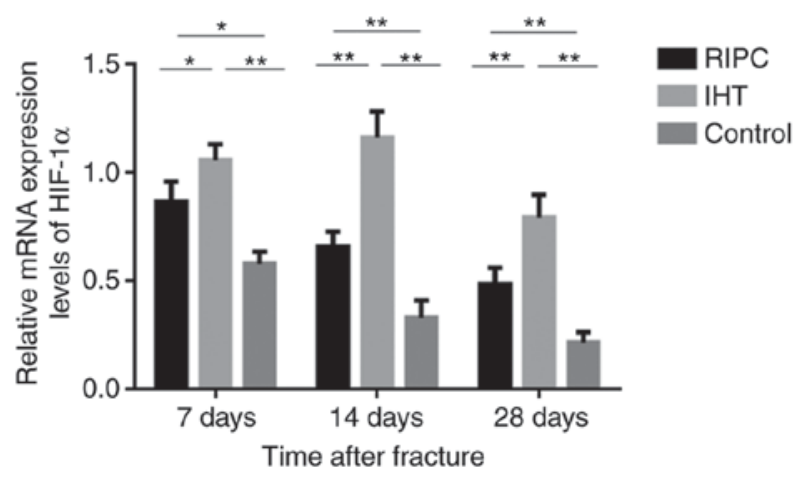

Figure 7. Protein and mRNA analysis of the expression of HIF-1 $\alpha$ in bone callus tissues of the IHT, RIPC and control groups. RIPC and IHT treatment upregulated the expression of HIF-1 $\alpha$. Furthermore, the expression levels of HIF-1 $\alpha$ in the IHT group were significantly increased compared with those in the RIPC group. (A) Western blot analysis of the protein expression of HIF-1 $\alpha$ in bone callus of IHT, RIPC and control group. (B) mRNA analysis of the expression of HIF-1 $\alpha$ in bone callus of the IHT group, RIPC group and control group. GAPDH was used as an internal control. Values are presented as the mean \pm standard deviation. ${ }^{*} \mathrm{P}<0.05$ and ${ }^{* * *} \mathrm{P}<0.01$. IHT, intermittent hypoxia training; RIPC, remote ischemic preconditioning; HIF-1 $\alpha$, hypoxia-inducible factor-1 $\alpha$.

Various IHT protocols, considering the number of hypoxic episodes, severity and total exposure duration, have been implemented, and different combinations have resulted in various responses (21). Accumulating evidence suggests that 'low dose' IHT may be a simple, safe and effective treatment, with considerable therapeutic potential for multiple clinical disorders (22). It has been reported that modest hypoxia (9-16\% inspired $\mathrm{O}_{2}$ ) and low cycle numbers (3-15 episodes per day) most often lead to beneficial effects without pathological effects, whereas severe hypoxia (2-8\% inspired $\mathrm{O}_{2}$ ) and an increased number of episodes per day (48-2,400 episodes/day) elicit progressive pathological effects (23). The IHT regimen of $5 \mathrm{~min}$, with $12 \% \mathrm{O}_{2}, 5$-min breaks and five cycles per day, used in the present study, may be the most effective. Previous studies have suggested that the clinical application of RIPC may be complex $(24,25)$. Although transient limb ischemia has been applied as a remote conditioning stimulus in various clinical settings (26), there is limited data regarding the optimization of RIPC protocols. The majority of investigations involving RIPC have been exploratory investigations and based on clinical experiences.

In conclusion, the present study demonstrated that RIPC and IHT at defined doses are efficient strategies to enhance fracture healing, which may function by upregulation of the expression of HIF-1 $\alpha$. Compared with RIPC, IHT more efficiently promoted bone formation and guiding of a rapid fracture healing course. Therefore, IHT may be an optimal choice for fracture patients, and requires further investigation.

\section{Acknowledgements}

The authors give their thanks to the research platform of Central Laboratory of Xuanwu Hospital Capital medical university (Beijing, China).

\section{Funding}

The present study was supported by the National Natural Science Foundation of China (grant no. 81541135) and Capital's Funds for Health Improvement and Research (grant no. 2018-2-2012).

\section{Availability of data and materials}

The datasets used and/or analyzed during the present study are available from the corresponding author on reasonable request. 


\section{Authors' contributions}

JQ performed most of the experiments and manuscript preparation. GG and JR performed the RT-qPCR analysis and western blotting. GC and ZL analyzed the data. MZ, SL and HS designed the experiments. All authors read and approved the final manuscript.

\section{Ethics approval and consent to participate}

The present study was approved by the Ethics Committee of Xuanwu Hospital.

\section{Patient consent for publication}

Not applicable.

\section{Competing interests}

The authors declare that they have no competing interests.

\section{References}

1. Tarantino U, Saturnino L, Scialdoni A, Feola M, Liuni FM, Tempesta V and Pistillo P: Fracture healing in elderly patients: New challenges for antiosteoporotic drugs. Aging Clin Exp Res 25 (Suppl 1): S105-S108, 2013.

2. Bliuc D, Nguyen ND, Alarkawi D, Nguyen TV, Eisman JA and Center JR: Accelerated bone loss and increased post-fracture mortality in elderly women and men. Osteoporos Int 26: $1331-1339,2015$

3. Randhawa PK, Bali A and Jaggi AS: RIPC for multiorgan salvage in clinical settings: Evolution of concept, evidences and mechanisms. Eur J Pharmacol 746: 317-332, 2015.

4. Zhou M, Lu S, Lu G, Huang J, Liu L, An S, Li Z and Shen H: Effects of remote ischemic postconditioning on fracture healing in rats. Mol Med Rep 15: 3186-3192, 2017.

5. Gabriele P, Ozello F, Tseroni V, Madon E and Ragona R: Interstitial hyperthermia (IHT): Technical problems and methodology. Adv Exp Med Biol 267: 121-127, 1990.

6. Korkushko OV, Shatilo VB and Ishchuk VA: Effectiveness of intermittent normabaric hypoxic trainings in elderly patients with coronary artery disease. Adv Gerontol 23: 476-482, 2010 (In Russian).

7. Yuasa M, Mignemi NA, Barnett JV, Cates JM, Nyman JS, Okawa A, Yoshii T, Schwartz HS, Stutz CM and Schoenecker JG: The temporal and spatial development of vascularity in a healing displaced fracture. Bone 67: 208-221, 2014.

8. Hovens IB, Schoemaker RG, van der Zee EA, Heineman E, Nyakas C and van Leeuwen BL: Surgery-induced behavioral changes in aged rats. Exp Gerontol 48: 1204-1211, 2013

9. Zhang L, Yi L, Chopp M, Kramer BC, Romanko M, Gosiewska A and Hong K: Intravenous administration of human umbilical tissue-derived cells improves neurological function in aged rats after embolic stroke. Cell Transplant 22: 1569-1576, 2013.
10. Livak KJ and Schmittgen TD: Analysis of relative gene expression data using real-time quantitative PCR and the 2(-Delta Delta C(T)) method. Methods 25: 402-408, 2001.

11. Tinubu J and Scalea TM: Management of fractures in a geriatric surgical patient. Surg Clin North Am 95: 115-128, 2015.

12. Hefner G, Stieffenhofer V, Gabriel S, Palmer G, Müller KM, Röschke J and Hiemke C: Side effects related to potentially inappropriate medications in elderly psychiatric patients under everyday pharmacotherapy. Eur J Clin Pharmacol 71: 165-172, 2015.

13. Peyrin F, Dong P, Pacureanu A and Langer M: Micro- and nano-CT for the study of bone ultrastructure. Curr Osteoporos Rep 12: 465-474, 2014.

14. O'Neill KR, Stutz CM, Mignemi NA, Burns MC, Murry MR, Nyman JS and Schoenecker JG: Micro-computed tomography assessment of the progression of fracture healing in mice. Bone 50: 1357-1367, 2012.

15. Oftadeh R, Perez-Viloria M, Villa-Camacho JC, Vaziri A and Nazarian A: Biomechanics and mechanobiology of trabecular bone: A review. J Biomech Eng: 137, 2015. doi: 10.1115/1.4029176.

16. Einhorn TA and Gerstenfeld LC: Fracture healing: Mechanisms and interventions. Nat Rev Rheumatol 11: 45-54, 2015.

17. Komori T: Regulation of osteoblast differentiation by transcription factors. J Cell Biochem 99: 1233-1239, 2006.

18. Keramaris NC, Calori GM, Nikolaou VS, Schemitsch EH and Giannoudis PV: Fracture vascularity and bone healing: A systematic review of the role of VEGF. Injury 39 (Suppl 2): S45-S57, 2008.

19. Kalakech H, Tamareille S, Pons S, Godin-Ribuot D, Carmeliet $P$, Furber A, Martin V, Berdeaux A, Ghaleh B and Prunier F: Role of hypoxia inducible factor-1alpha in remote limb ischemic preconditioning. J Mol Cell Cardiol 65: 98-104, 2013.

20. Maes C, Carmeliet G and Schipani E: Hypoxia-driven pathways in bone development, regeneration and disease. Nat Rev Rheumatol 8: 358-366, 2012.

21. Serebrovskaya TV, Nosar VI, Bratus LV, Gavenauskas BL and Mankovska IM: Tissue oxygenation and mitochondrial respiration under different modes of intermittent hypoxia. High Alt Med Biol 14: 280-288, 2013.

22. Serebrovska TV, Serebrovska ZO and Egorov E: Fitness and therapeutic potential of intermittent hypoxia training: A matter of dose. Fiziol Zh 62: 78-91, 2016.

23. Navarrete-Opazo A and Mitchell GS: Therapeutic potential of intermittent hypoxia: A matter of dose. Am J Physiol Regul Integr Comp Physiol 307: R1181-R1197, 2014.

24. Anttila V, Haapanen H, Yannopoulos F, Herajarvi J, Anttila T and Juvonen T: Review of remote ischemic preconditioning: From laboratory studies to clinical trials. Scand Cardiovasc J 50: 355-361, 2016.

25. Martin-Puig S, Tello D and Aragonés J: Novel perspectives on the PHD-HIF oxygen sensing pathway in cardioprotection mediated by IPC and RIPC. Front Physiol 6: 137, 2015.

26. Candilio L, Malik A, Ariti C, Barnard M, Di Salvo C, Lawrence D, Hayward M, Yap J, Roberts N, Sheikh A, et al: Effect of remote ischaemic preconditioning on clinical outcomes in patients undergoing cardiac bypass surgery: A randomised controlled clinical trial. Heart 101: 185-192, 2015. 